\title{
Cerebral connectivity and psychotic personality traits
}

\section{A diffusion tensor imaging study}

Received: 7 December 2006 / Accepted: 29 November 2007 / Published online: 23 February 2008

Abstract This study aims to investigate the relationship between regional connectivity in the brain white matter and the presence of psychotic personality traits, in healthy subjects with psychotic traits. Thirteen healthy controls were administered the MMPI-2, to assess psychotic traits and, according to MMPI results, a dichotomization into a group of "high-psychotic" and "low-psychotic" was performed. Diffusion tensor imaging (DTI) was used as a non-invasive measure, in order to obtain information about the fractional anisotropy (FA), an intravoxel index of local connectivity and, by means of a voxelwise approach, the between-group differences of

Umberto Volpe and Andrea Federspiel contributed equally to this work.

U. Volpe $\cdot$ A. Mucci $\cdot$ S. Galderisi $\cdot$ M. Maj

Department of Psychiatry

University of Naples SUN

Naples, Italy

A. Federspiel $(\bowtie) \cdot T$. Dierks

Department of Psychiatric Neurophysiology

University Hospital of Psychiatry

University of Bern

Bolligenstrasse 111

3000 Bern 60, Switzerland

Tel.: +41-31/9309371

Fax: $+41-31 / 9309961$

E-Mail: federspiel@puk.unibe.ch

A. Frank · L.-O. Wahlund - U. Volpe $\cdot$ A. Federspiel

T. Dierks

Department of Clinical Neuroscience, Occupational Therapy and Elderly Care Research (Neurotec)

Division of Clinical Geriatrics

Karolinska Institute

Huddinge University Hospital

Stockholm, Sweden

\section{A. Frank}

$\precsim$ Department of Hospital Physics

z Karolinska Institute

Huddinge University Hospital

Stockholm, Sweden the FA values were calculated. The "high-psychotic" group showed higher FA in the left arcuate fasciculus. Subjects with low scores for psychotic traits had significantly higher FA in the corpus callosum, right arcuate fasciculus, and fronto-parietal fibers. In line with previous brain imaging studies of schizophrenia spectrum disorders, our results suggest that psychotic personality traits are related to altered connectivity and brain asymmetry.

Key words brain connectivity - brain asymmetry · diffusion tensor imaging (DTI) - fractional anisotropy - personality assessment - psychotic personality traits . psychosis $\cdot$ schizophrenia spectrum

\section{Introduction}

Schizophrenic psychoses have long been claimed to be associated with subtle brain abnormalities, although no pathognomonic lesion has been definitively associated to the functional deficits observed in these patients. Several neuropsychophysiological models of schizophrenia describe this illness as the result of a dysfunctional integration among neural systems: more in detail, it has been postulated that schizophrenic psychoses arise from a defective modulation of associative changes in the neural systems implicated in emotion, memory and learning [16]. Imaging connections among brain areas could contribute to the validation of these models.

Since the first descriptions of schizophrenia by Kraepelin [25] and Bleuler [6], a relationship between a "schizoid" personality and the development of a future psychosis has been proposed. According to Kretschmer [26], a "schizoid temperament" exists on a continuum, ranging from psychotic personality traits in otherwise healthy subjects to manifest psy- 
chosis. Since then, most of the studies have demonstrated genetic, psychopathological, neurobiological associations between cluster A personality disorders and psychosis, resulting in the "neurodevelopmental hypothesis" for the so-called "schizophrenia spectrum" [30]. Further support to this theory comes from epidemiological studies on the topic: categorical operationalized diagnostic formulations of the schizophrenic syndrome describe it as a nosological entity mostly shaped by an exclusion process, whose blurred diagnostic boundaries reflect the lack of an underlying paradigm [28]; instead, epidemiological surveys in the general population (for a review see [21]) tended to show that schizophrenia does not seem to be a discrete illness entity but could be better described as a psychopathological extreme of a "psychotic spectrum", ranging from normal experiences and behaviours to severe psychotic symptoms. A recent 20-year longitudinal study [35], aimed to investigate the prevalence and the course of psychotic experiences in a large sample of healthy subjects from the general population, confirmed that psychotic phenomena are better conceived as a dimension which stretches from the normal/subclinical to the clinical level and claimed for further research to investigate the pathways which may lead from psychosis proneness to a clinical psychotic disorder.

The neurobiological investigation of psychometrically identified psychosis-prone subjects (i.e., subjects with high scores on scales assessing psychotic traits or experiences) has confirmed the presence of psychophysiological abnormalities akin to those seen in schizophrenia spectrum disorders [31].

Diffusion tensor imaging (DTI) is a magnetic resonance imaging (MRI) technique, which evaluates the motion of water molecules within a biological tissue at the microscopic level. This principle has been applied to the investigation of the diffusion-driven displacement of the molecules of water in the human brain white matter, allowing to detect the preferential movement of water molecules within the boundaries of the fibers, due to their anisotropic properties (i.e., the amount of anisotropy correlates with the directionality and the coherence of molecular mobility in the fiber tracts); this technique can be useful to detect the microstructure of different brain areas and their connections.

Previous studies, investigating white matter abnormalities in schizophrenic subjects with DTI, reported white matter loss of orientation and organization in specific brain areas and several disruptions of commissural connectivity, which mostly consisted of decreased values of fractional anisotropy (FA) in intra-hemispheric (arcuate, cingulate and uncinate fasciculi) and inter-hemispheric fibers (corpus callosum) (for a review, see [23]). There is recent evidence that fronto-parieto-temporal misconnection might be particularly important in schizophrenia [17, 36]. Furthermore, several DTI findings converged towards a disruption of white matter organization in frontal areas in schizophrenia, although a definitive conclusion has not been achieved yet [23]. Recently, DTI techniques have been recognized as useful to extend disconnectionist paradigms even beyond schizophrenia and to neurodevelopmental, neurodegenerative and subtle cognitive disorders [10].

The aim of the present study was to evaluate the brain connectivity patterns, investigated by means of a DTI technique with a voxel-based approach, in relationship to psychotic personality traits, in a sample of healthy subjects. Our working hypothesis was that there could be a relationship between different brain connectivity patterns and the presence of psychotic personality traits, which might represent a sub-clinical entity within the schizophrenia spectrum.

\section{Materials and methods}

\section{Experimental sample}

Thirteen volunteers ( 8 females and 5 males) were recruited among staff members and students of the Karolinska Institute of Stockholm (Sweden). All asked subjects accepted to participate in the study, gave their informed consent to experimental procedures and were included in the final experimental sample. All subjects were right-handed, as assessed by the Edinburgh Inventory [33], their mean age was 29.7 years ( $\mathrm{SD}=4.6$ years) and their mean education was 20.4 years $(S D=3.9$ ). After a one-hour clinical interview with a skilled psychiatrist (U.V.), none of them resulted to have personal and family history of major medical diseases and none had Axis I or Axis II DSM-IV diagnoses. Subjects were not on psychotropic or any other medication and did not report alcohol or other substances abuse. All participants had normal structural MRI and gave their informed written consent to participate in the study. The local ethical committee approved the study procedures.

\section{Personality assessment}

In order to assess the psychotic personality traits, the Minnesota Multiphasic Personality Inventory (MMPI-2) was chosen because it is widely considered a reliable measure of both personality characteristics and clinical status [7, 9, 11, 18, 27, 38]. High scores on the MMPI-2 Schizophrenia ( $\mathrm{Sc}$ ), Paranoia $(\mathrm{Pa})$ and Psychopathic Deviate (Pd) scales have been previously reported in remitted schizophrenic patients [37] and in non schizophrenic first-degree relatives of schizophrenic patients [1], suggesting that these scales may be indicators of vulnerability factors for schizophrenia.

The full version of the MMPI-2 was administered to all subjects by the same researcher and all subjects correctly completed the test. Subjects' MMPI-2 profile validity was established by means of the Variable Response Inconsistency Scale, with a cut-off score of ten. $\mathrm{K}$-corrected T-scores with adult norms were used in the scoring procedure to allow comparison with other studies [5]. A mediansplit method was used on the MMPI-2 Sc, Pa and Pd scales to categorize subjects in two groups: "high-psychotic" subjects (those who had high standardized T-scores for psychotic traits) and "lowpsychotic" subjects (those who had low standardized T-scores for psychotic traits).

\section{MRI/DTI recording}

All images were acquired using a $1.5 \mathrm{~T}$ whole body MRI system (Siemens Vision, Erlangen, Germany), equipped with a standard radio frequency head coil. Head motion was minimized within the 
coil using a vacuum deflatable pillow and an open-cell polyurethane foam.

The MRI session included the acquisition of a set of 3D T1weighted (Magnetization Prepared Rapid Acquisition Gradient Echo, MP-RAGE) images, providing 170 sagittal slices with TR $=11.4 \mathrm{~ms}, \mathrm{TE}=4.4 \mathrm{~ms}$, flip angle $=15^{\circ}$, thickness $=1.0 \mathrm{~mm}$, field of view $(\mathrm{FOV})=256 \mathrm{~mm} \times 256 \mathrm{~mm}$, and matrix size $=256$ $\times 256$. The voxel resolution was $1 \mathrm{~mm} \times 1 \mathrm{~mm} \times 1 \mathrm{~mm}$.

The diffusion-weighted images were acquired using a singleshot spin-echo echo-planar imaging (SE-EPI) sequence. The gradient amplitudes and duration have to be chosen, such that tissue dependent diffusion coefficients can be detected by the signal attenuation. In our experiment we used $G=15 \mathrm{mT} / \mathrm{m}$, intergradient time interval $25.0 \mathrm{~ms}$. The diffusion sensitizing gradients were applied on two axes simultaneously around the $180^{\circ}$ pulse at $b=1,014 \mathrm{~s} / \mathrm{mm}^{2} /$ axis along six noncollinear directions:

$G_{x}, G_{y}, G_{z}=[(1,1,0),(0,1,1),(1,0,1),(-1,1,0), .(0,1,-1),(1,0,-1)]$.

The calculation and diagonalization of the diffusion tensor was based on the multivariate regression approach [2]. The imaging parameters of our single-shot spin-echo echo-planar imaging (SE-EPI) sequence were chosen as follows: Matrix $128 \times 128$, TE $=123 \mathrm{~ms}$, FOV $=240 \mathrm{~mm} \times 240 \mathrm{~mm}$, slice thickness $5 \mathrm{~mm}, 12$ axial continuous slices, $\mathrm{TR}=3 \mathrm{~s}$, pixel bandwidth $\mathrm{BW}=$ $1.25 \mathrm{kHz}$, standard head coil, head-neck standard shimming was performed. The voxel resolution was $1.875 \mathrm{~mm} \times 1.875 \mathrm{~mm} \times 5$ $\mathrm{mm}$. Eddy-current corrections were included. Additionally, one image was acquired with no gradients applied $\left(b=0 \mathrm{~s} / \mathrm{mm}^{2}\right)$.

\section{DTI processing and analysis}

Automatic segmentation (BrainVoyager QX 1.4; Brain Innovation, Maastricht, Netherlands) of the 3D anatomical images (standardized Talairach space) for each subject yielded a white matter map. This map was used to mask the FA maps in order to restrict the statistical computation only on white matter regions. The individual standardized 3D white matter maps were used to compute the largest possible 3D white matter-template (Fig. 1). Six independent elements of the diffusion tensor were extracted [2,3]. Eigenvalues (magnitude) and eigenvectors (direction) were determined for each voxel, and the FA maps were constructed.

Co-registration of the 2D FA maps to the 3D structural images was manually performed using the scanner's slice position parameters of the SE-EPI measurements and the T1-weighted anatomical measurements. The quality of this co-registration step was checked using spm5 (Wellcome Department of Imaging Neuroscience, London, England; http://www.fil.ion.ucl.ac.uk) in the following way: (1) we extracted the co-registered files and the raw files to analyze format, (2) then, by means of the spm5 software, we performed the co-registration step, (3) within spm5, we the used the ImCalc module to compute the difference between the manually co-registered files and the spm5 co-registered files in order to statistically
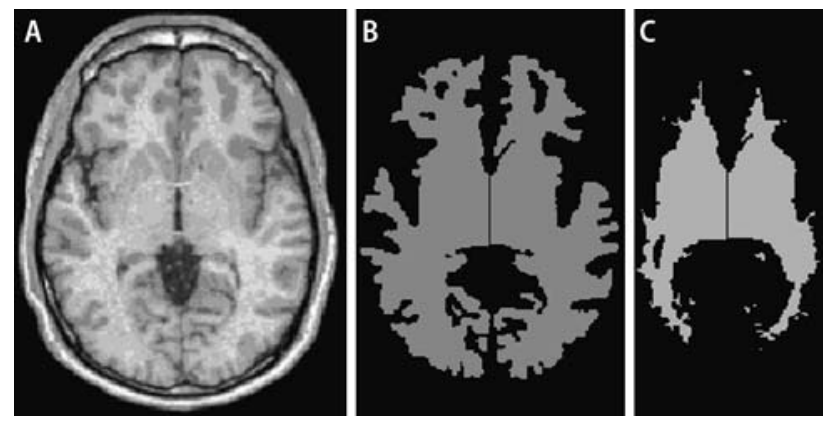

Fig. 1 DTI "masking": normalized anatomical 3D images (a) were used to calculate white matter map for each subject (b); then, each map was used to build the largest possible 3D white matter-template for the fractional anisotropy (FA) map (c) check for any differences between these files, (4) then we performed a one-sample t-test with these difference images and we did find no significant difference between manually- and mathematically coregistered procedure was found $[t(94)=1.034 ; P=0.3]$.

The Talairach transformation is performed on the anatomical dataset and, in a subsequent step, the transformation matrix found on the anatomical data is applied to the FA maps. During this coregistration the voxel dimension of the FA maps was interpolated to $1.0 \mathrm{~mm} \times 1.0 \mathrm{~mm} \times 1.0 \mathrm{~mm}$. Visual inspection of all maps for each subject suggested no need for additional susceptibility artifact correction. Finally, the images were smoothed using a Gaussian filter with a FWHM of $7.5 \mathrm{~mm}$. This filter size was chosen for the following reasons: we identified the average FA values of background to be in range of 0.1 . The background was defined as values within the gray matter and within cerebrospinal fluid (CSF) of each subject. The mean FA values that we identified as signal were in the range of 0.4 and were extracted from white matter of each subject. From these values we estimated that our signal is approximately four times the background $(S N R \approx 4)$. Therefore we used a Gaussian Kernel with FWHM of $7.5 \mathrm{~mm}$, which are four times the voxel dimensions of the raw data [22].

In the definition of our SNR we assume that the noise within the white matter can be expressed by the FA values of the gray matter and especially of CSF, i.e., in a region of low diffusivity (gray matter), and in region with long T1 and long T2 (CSF). Furthermore, we assume that the noise extracted within these regions has the characteristics of "white noise".

Comparisons of FA maps were subsequently computed on a voxel-by-voxel basis, by means of homemade scripts, written in Matlab (ver. 6.5, r. 13, Mathworks Inc., 2002), since this option was not present within Brainvoyager QX.

In order to evaluate differences between FA values of the "Lowpsychotic" and the "High-psychotic" group, a general linear model (GLM) analysis was computed for each voxel within the 3D white matter template. To identify the most significant regions, "clusters" were defined as 60 or more neighboring voxels $\left(60 \mathrm{~mm}^{3}\right)$ exceeding the statistical value of $2.178(P<.05)$. Clusters were assigned to the underlying white matter using $3 \mathrm{D}$ anatomical data.

Since the sex distribution in the two subgroups was not perfectly balanced, in order to exclude that the differences in regional FA were due to a sex effect, we also performed two linear regression

Table 1 Demographic and MMPI-2 characteristics

\begin{tabular}{|c|c|c|c|c|c|c|}
\hline \multirow[t]{2}{*}{ Sex } & \multirow{2}{*}{$\begin{array}{l}\text { Edu } \\
\text { (years) }\end{array}$} & \multirow{2}{*}{$\begin{array}{l}\text { Age } \\
\text { (years) }\end{array}$} & \multicolumn{3}{|c|}{$T$-scores } & \multirow[t]{2}{*}{ Group } \\
\hline & & & PA & PD & SCF & \\
\hline$t$-value & & & & & & \\
\hline $\begin{array}{c}2.11 \\
P \text {-value }\end{array}$ & 0.32 & 0.10 & 1.63 & 1.32 & 2.24 & \\
\hline 0.06 & 0.76 & 0.92 & 0.13 & 0.21 & 0.05 & \\
\hline $\mathrm{F}$ & 18 & 26 & 44 & 36 & 31 & Low \\
\hline $\mathrm{F}$ & 18 & 29 & 46 & 54 & 31 & Low \\
\hline $\mathrm{F}$ & 23 & 31 & 40 & 51 & 30 & Low \\
\hline $\mathrm{F}$ & 18 & 26 & 43 & 39 & 34 & Low \\
\hline $\mathrm{F}$ & 18 & 26 & 46 & 51 & 30 & Low \\
\hline M & 23 & 39 & 32 & 55 & 30 & Low \\
\hline $\mathrm{F}$ & 18 & 30 & 49 & 59 & 30 & Low \\
\hline M & 19 & 27 & 45 & 69 & 30 & High \\
\hline M & 19 & 28 & 49 & 44 & 46 & High \\
\hline $\mathrm{F}$ & 18 & 30 & 51 & 51 & 50 & High \\
\hline M & 22 & 31 & 53 & 55 & 58 & High \\
\hline M & 23 & 38 & 39 & 55 & 30 & High \\
\hline $\mathrm{F}$ & 18 & 25 & 49 & 59 & 32 & High \\
\hline
\end{tabular}

Edu educational level, $T$-scores MMPI-2 standardized scores, PA paranoia subscale, $P D$ psychopathic deviate subscale, SCF schizophrenia subscale; each individual, based on the median split method, has been categorized as belonging either to the group with high scores for psychotic traits or to the group with low scores for psychotic traits 
Table 2 Clusters of significant DTI and MMPI-2 correlation

\begin{tabular}{|c|c|c|c|c|c|c|c|c|c|c|c|c|c|c|c|}
\hline \multirow[t]{2}{*}{$\begin{array}{l}\text { \# Voxel } \\
\text { within } \\
\text { cluster }\end{array}$} & \multicolumn{3}{|c|}{$\begin{array}{l}\text { Talairach coordinates } \\
\text { at center of gravity } \\
\text { (mean) }\end{array}$} & \multicolumn{4}{|c|}{$\begin{array}{l}\text { Fractional anisotropy } \\
\text { (mean } \pm \text { standard deviation) }\end{array}$} & \multicolumn{2}{|c|}{ Statistics } & \multirow[t]{2}{*}{$\begin{array}{l}\text { Cerebral } \\
\text { lobe }\end{array}$} & \multirow[t]{2}{*}{$\begin{array}{l}\text { Hemi- } \\
\text { sphere }\end{array}$} & \multicolumn{2}{|c|}{ Reduced GLM } & \multicolumn{2}{|l|}{ Full GLM } \\
\hline & $x$ & $y$ & $z$ & $\begin{array}{l}\text { Low } \\
\text { (males) }\end{array}$ & $\begin{array}{l}\text { Low } \\
\text { (females) }\end{array}$ & $\begin{array}{l}\text { High } \\
\text { (males) }\end{array}$ & $\begin{array}{l}\text { High } \\
\text { (females) }\end{array}$ & $z$-value & $P$-value & & & $\begin{array}{l}\text { Explained } \\
\text { variance }\end{array}$ & $P$-value & $\begin{array}{l}\text { Explained } \\
\text { variance }\end{array}$ & value \\
\hline 25 & -37.0 & 27.5 & 14.7 & 0.2 & $0.19 \pm 0.06$ & $0.28 \pm 0.02$ & $0.29 \pm 0.03$ & 3.7228 & 0.0029 & Frontal & L & 0.59 & 0.0021 & 0.62 & .0246 \\
\hline 75 & -27.6 & 5.6 & 34.2 & 0.24 & $0.31 \pm 0.10$ & $0.52 \pm 0.05$ & $0.47 \pm 0.07$ & 4.4819 & 0.0007 & Frontal & L & 0.65 & 0.0008 & 0.66 & 0.0161 \\
\hline 521 & -31.9 & -17.2 & 25.1 & 0.32 & $0.38 \pm 0.12$ & $0.67 \pm 0.04$ & $0.55 \pm 0.06$ & 4.3193 & 0.001 & Frontal & L & 0.63 & 0.0011 & 0.66 & 0.0169 \\
\hline 409 & -16.5 & 7.5 & 28.7 & 0.6 & $0.48 \pm 0.07$ & $0.70 \pm 0.10$ & $0.77 \pm 0.09$ & 4.6019 & 0.0006 & Frontal & L & 0.7 & 0.0003 & 0.77 & 0.0027 \\
\hline 148 & -25.2 & 0.1 & 38.9 & 0.43 & $0.46 \pm 0.11$ & $0.19 \pm 0.13$ & $0.18 \pm 0.14$ & 4.1958 & 0.0012 & Frontal & L & 0.64 & 0.0009 & 0.64 & 0.02 \\
\hline 169 & -30.5 & 21.5 & 12.4 & 0.23 & $0.33 \pm 0.08$ & $0.50 \pm 0.06$ & $0.51 \pm 0.05$ & 4.8688 & 0.0004 & Limbic & L & 0.69 & 0.0003 & 0.74 & .0046 \\
\hline 610 & -29.9 & 1.6 & 19.7 & 0.44 & $0.32 \pm 0.08$ & $0.59 \pm 0.02$ & $0.57 \pm 0.06$ & 5.7609 & 0.0001 & Limbic & L & 0.78 & 0.0001 & 0.81 & .0011 \\
\hline 465 & -40.0 & -40.6 & -6.2 & 0.33 & $0.37 \pm 0.11$ & $0.69 \pm 0.08$ & $0.53 \pm 0.11$ & 3.7312 & 0.0029 & Limbic & L & 0.59 & 0.0021 & 0.7 & .0092 \\
\hline 262 & -18.9 & 6.1 & -4.2 & 0.3 & $0.42 \pm 0.09$ & $0.77 \pm 0.15$ & $0.59 \pm 0.05$ & 3.6395 & 0.0034 & & L & 0.61 & & 0.64 & .0195 \\
\hline 799 & -22.1 & -25.5 & 15.5 & 0.58 & $0.48 \pm 0.13$ & $0.83 \pm 0.03$ & $0.77 \pm 0.05$ & 5.0477 & 0.0003 & Limbic & L & 0.74 & 0.0001 & 0.78 & .0025 \\
\hline 251 & -2.3 & -14.5 & -7.0 & 0.64 & $0.58 \pm 0.12$ & $0.35 \pm 0.15$ & $0.37 \pm 0.12$ & 3.3631 & 0.0056 & Limbic & L & 0.49 & 0.0068 & 0.5 & 0.0849 \\
\hline 2,732 & -8.0 & -6.1 & 29.9 & 0.83 & $0.69 \pm 0.04$ & $0.40 \pm 0.16$ & $0.39 \pm 0.20$ & 3.6085 & 0.0036 & imbic & L & 0.67 & 0.0005 & 0.69 & 0.0112 \\
\hline 230 & -33.7 & -59.4 & 9.2 & 0.28 & $0.26 \pm 0.06$ & $0.48 \pm 0.09$ & $0.45 \pm 0.14$ & 3.5219 & 0.0042 & Occipital & L & 0.63 & 0.0011 & 0.64 & 0.0214 \\
\hline 44 & -30.9 & -65.6 & 7.8 & 0.3 & $0.29 \pm 0.09$ & $0.47 \pm 0.07$ & $0.47 \pm 0.14$ & 3.3928 & 53 & & L & & 21 & 0.65 & .0179 \\
\hline 93 & -5.1 & -83.1 & 7.2 & 0.55 & $0.45 \pm 0.15$ & $0.19 \pm 0.14$ & $0.16 \pm 0.16$ & 3.6289 & 35 & ital & L & & 36 & 0.56 & .0479 \\
\hline 43 & -32.9 & -28.4 & 39.2 & 0.41 & $0.35 \pm 0.17$ & $0.13 \pm 0.11$ & $0.11 \pm 0.10$ & 3.1779 & 0.008 & Parietal & L & 0.47 & 087 & .5 & 0827 \\
\hline 516 & -9.9 & -47.1 & 23.8 & 0.56 & $0.66 \pm 0.10$ & $0.31 \pm 0.06$ & $0.44 \pm 0.06$ & 5.0534 & 0.0003 & Parietal & L & 0.7 & & 2 & .001 \\
\hline 69 & -37.1 & -57.5 & 19.3 & 0.24 & $0.22 \pm 0.10$ & $0.39 \pm 0.09$ & $0.37=$ & 3.2743 & 0.0067 & Ten & L & 0 & 0.0065 & 0.59 & .0367 \\
\hline 167 & -43.2 & -20.2 & -2.8 & 0.32 & $0.36 \pm 0.04$ & $0.20 \pm 0.05$ & $0.24 \pm 0.04$ & 4.7377 & 0.0005 & Temporal & L & 0.70 & 0.0003 & 0.76 & .0034 \\
\hline 541 & 52.8 & -26.1 & 6.7 & 0.38 & $0.40 \pm 0.06$ & $0.25 \pm 0.04$ & $0.19 \pm 0.03$ & 6.2689 & 0.00001 & Temporal & L & 0.79 & 0.0001 & 0.82 & 0.0008 \\
\hline 32 & 44.4 & -9.0 & -17.4 & 0.42 & $0.44 \pm 0.10$ & $0.23 \pm 0.04$ & $0.34 \pm 0.06$ & 3.2174 & 0.0074 & Temporal & L & 0.48 & 0.0078 & 0.62 & 0.0249 \\
\hline 57 & 25.9 & 30.7 & 0.2 & 0.36 & $0.45 \pm 0.08$ & $0.63 \pm 0.05$ & $0.57 \pm 0.03$ & 4.2272 & 0.0012 & Frontal & $\mathrm{R}$ & 0 & 0.0012 & 0.65 & 0.0191 \\
\hline 37 & 2 & 25.8 & -8.8 & ( & 12 & $0.55=$ & 0.6 & & & & $\mathrm{R}$ & & & 0.6 & 339 \\
\hline 3,00 & 11.0 & -0.2 & 21.8 & 0.41 & 0.37 & 0.72 & 0.67 & 7.4 & 0.0 & Frc & $\mathrm{R}$ & 0 & 01 & 0.86 & 03 \\
\hline 44 & 46.0 & 18.6 & 15.2 & 0.3 & 0.07 & 0.19 & 0.20 & 3.9634 & 0.0019 & & $\mathrm{R}$ & 0 & 36 & 0 & 0537 \\
\hline 152 & 54.5 & -16.8 & 21.3 & 0.4 & 0.03 & 0.20 & 0.17 & 4.8403 & 0.0 & & $\mathrm{R}$ & 0. & 001 & 0.7 & .0019 \\
\hline 72 & 40.8 & -8.0 & 36.9 & 0.49 & $0.37 \pm 0.13$ & $0.18 \pm 0.04$ & $0.20 \pm 0.09$ & 3.4487 & 0.0048 & Frontal & $\mathrm{R}$ & & 0.0049 & 0.53 & .0639 \\
\hline 114 & 24.7 & 43.6 & 3.1 & 0.51 & $0.43 \pm 0.08$ & $0.27 \pm 0.06$ & $0.20 \pm 0.17$ & 3.2412 & 0.0071 & Frontal & $\mathrm{R}$ & 0.55 & 0.0036 & 0.59 & 0.0346 \\
\hline 77 & 25.9 & 26.3 & 15.2 & 0.59 & $0.56 \pm 0.09$ & $0.40 \pm 0.02$ & $0.42 \pm 0.03$ & 3.92 & 0.002 & Frontal & $\mathrm{R}$ & 0.63 & 0.001 & 0.66 & 0.0157 \\
\hline 667 & 33.8 & 3.7 & 22.8 & 0.67 & $0.64 \pm 0.04$ & $0.37 \pm 0.05$ & $0.36 \pm 0.11$ & 5.5607 & 0.0001 & Frontal & $\mathrm{R}$ & 0.86 & 0.0001 & 0.87 & 0.0002 \\
\hline 283 & 31.6 & -10.3 & 29.3 & 0.8 & 0.14 & $0.39 \pm 0.15$ & $0.45 \pm 0.09$ & 3.5661 & & & $\mathrm{R}$ & & & 0.55 & 0.0523 \\
\hline 348 & 37.0 & 20.0 & 21.1 & & 6 & 0.2 & & & & & $\mathrm{R}$ & & & 0 & 83 \\
\hline 44 & 35.6 & 0 & 34.2 & 0.4 & 11 & $0.33 \pm$ & 0.27 & 3.5 & 0.0 & & $\mathrm{R}$ & 0 & 03 & 0. & 82 \\
\hline 47 & 17.0 & -0.5 & 3.8 & 0.5 & 0.14 & $0.76 \pm 0.16$ & 0.78 & 3.4765 & 0.0046 & bic & $\mathrm{R}$ & 0 & 0 & 0.5 & .0444 \\
\hline 1,91 & 31.5 & -8.3 & -4.2 & 0.61 & $0.53 \pm 0.10$ & $0.30 \pm 0.05$ & $0.29 \pm 0.08$ & 5.1898 & 02 & ic & $\mathrm{R}$ & 0.72 & 0.0002 & 0.76 & 0.0032 \\
\hline 86 & 39.3 & -4.7 & 21.6 & 0.5 & $0.57 \pm 0.11$ & $0.31 \pm 0.04$ & $0.39 \pm 0.06$ & 4.0923 & 0.0015 & Limbic & $\mathrm{R}$ & 0.6 & 0.0016 & 0.7 & 0.0087 \\
\hline 74 & 6.8 & -77.0 & 11.1 & 0.29 & $0.26 \pm 0.07$ & $0.43 \pm 0.10$ & $0.42 \pm 0.08$ & 3.6297 & 0.0035 & Occipital & $\mathrm{R}$ & 0.58 & 0.0023 & 0.59 & 0.0378 \\
\hline 497 & 12.0 & -66.3 & 0.4 & 0.45 & $0.30 \pm 0.09$ & $0.53 \pm 0.05$ & $0.50 \pm 0.11$ & 3.886 & 0.0022 & Occipital & $\mathrm{R}$ & 0.58 & 0.0024 & 0.68 & 0.0116 \\
\hline 101 & 14.3 & -80.5 & -7.1 & 0.34 & $0.35 \pm 0.11$ & $0.54 \pm 0.02$ & $0.52 \pm 0.08$ & 4.0146 & 0.0017 & & $\mathrm{R}$ & 0.60 & 0.0016 & 0.61 & 0.0287 \\
\hline 27 & 26.2 & -76.7 & -7.9 & 0.34 & $9 \pm 0.09$ & $0.20 \pm 0.10$ & $0.27 \pm 0.05$ & 3.2544 & & & $\mathrm{R}$ & 0. & & 0.57 & 0.0454 \\
\hline 251 & 46.7 & -53.4 & -5.5 & 0.29 & $0.40 \pm 0.13$ & $0.21 \pm 0.10$ & $0.17 \pm 0.09$ & 3.3234 & 0.0061 & & $\mathrm{R}$ & 0.5 & 0.0048 & 0.53 & 0.0622 \\
\hline 231 & 39.1 & -63.7 & 5.2 & 0.43 & $0.39 \pm 0.12$ & $0.20 \pm 0.13$ & $0.18 \pm 0.10$ & 3.3986 & 0.0053 & ital & $\mathrm{R}$ & 0. & 52 & 0.55 & 0.0542 \\
\hline 146 & 23.1 & -32.5 & -7.0 & 0.24 & $0.43 \pm 0.15$ & $0.62 \pm 0.04$ & $0.78 \pm 0.15$ & 3.6938 & 0.0031 & Parietal & $\mathrm{R}$ & 0. & 0.0033 & 0.69 & 0.0112 \\
\hline 71 & 17.3 & -66.2 & 35.5 & 0.41 & $0.36 \pm 0.11$ & $0.19 \pm 0.02$ & $0.17 \pm 0.10$ & 3.9213 & 0.0 & & $\mathrm{R}$ & 0.5 & 0.0022 & 0.62 & 0.0271 \\
\hline 90 & 30.1 & -69.1 & 17.2 & 0.24 & $0.47 \pm 0.12$ & $0.26 \pm 0.05$ & $0.21 \pm 0.09$ & 3.2518 & 0.0069 & Parietal & $\mathrm{R}$ & 0.49 & 0.007 & 0.55 & 0.0542 \\
\hline 32 & 29.4 & -15.7 & 38.1 & 0.68 & $0.60 \pm 0.20$ & $0.37 \pm 0.13$ & $0.27 \pm 0.15$ & 3.2125 & 0.0075 & Parietal & $\mathrm{R}$ & 0.48 & 0.0083 & 0.54 & 0.0602 \\
\hline 44 & 26.5 & -36.7 & 34.6 & 0.58 & $0.63 \pm 0.07$ & $0.41 \pm 0.10$ & $0.41 \pm 0.09$ & 4.486 & 0.0007 & Parietal & $\mathrm{R}$ & 0.54 & 0.0041 & 0.56 & 0.0491 \\
\hline 1,070 & 42.0 & -18.2 & 29.2 & 0.48 & $0.53 \pm 0.10$ & $0.29 \pm 0.04$ & $0.27 \pm 0.09$ & 5.2197 & 0.0002 & Parietal & $\mathrm{R}$ & 0.72 & 0.0002 & 0.72 & 0.0068 \\
\hline 127 & 16.7 & -17.2 & 9.7 & 0.73 & $0.54 \pm 0.11$ & $0.81 \pm 0.04$ & $0.81 \pm 0.11$ & 4.1008 & 0.0015 & Temporal & $\mathrm{R}$ & 0.61 & 0.0014 & 0.66 & 0.0151 \\
\hline 330 & 30.6 & -25.2 & -14.5 & 0.36 & $0.47 \pm 0.19$ & $0.76 \pm 0.09$ & $0.69 \pm 0.10$ & 3.238 & 0.0071 & Temporal & $\mathrm{R}$ & 0.49 & 0.0074 & 0.53 & 0.0646 \\
\hline 1,083 & 43.9 & -35.4 & -7.5 & 0.68 & $0.59 \pm 0.10$ & $0.35 \pm 0.01$ & $0.33 \pm 0.05$ & 5.2646 & 0.0002 & Temporal & $\mathrm{R}$ & 0.77 & 0.0001 & 0.79 & 0.0017 \\
\hline
\end{tabular}

Table containing each cluster with FA values, Talairach coordinates of the centers of gravity, separated for male and female subjects and for group with high and low scores for psychotic traits and including the $z$ - and $P$-value of the GLM statistics for group comparison. Furthermore, the table contains for each cluster the results of the linear regression analysis with two models: (a) reduced GLM (here we assumed that MMPI-2 Sc, Pa and Pd scales values were the only predictors for FA response) and (b) full GLM (here we assumed that the FA may not only be predicted by MMPI-2 Sc, Pa and Pd scales, but also by sex and age). The explained variances of these GLM models are expressed by the adjusted $R$-square

analyses within the framework of GLM. Two models were constructed: in the first model, we assumed that MMPI-2 Sc, Pa and Pd scales values were the only predictors for FA response (Reduced GLM model); in the second model, we assumed that the FA may not only be predicted by MMPI-2 Sc, Pa and Pd scales, but also by sex and age (Full GLM model). The explained variances of these GLM models were expressed by the adjusted $R$-Square values. Finally, to test the goodness of fit between these two models we used an F-test 
with the sum of the square of the residuals of the reduced (Rss reduced) and full GLM (Rss full), with the following parameters ( $P$ reduced $=3 ; q$ full $=5 ; n=13)$ :

$$
\begin{aligned}
F= & {\left[\left(R s s_{\text {reduced }}-R s s_{\text {full }}\right)\left(q_{\text {full }}-P_{\text {reduced }}\right)^{-1}\right] } \\
& \times\left[R s s_{\text {full }}\left(n-q_{\text {full }}\right)^{-1}\right]^{-1} \\
\approx & F_{q-p, n-q}^{(\alpha)} .
\end{aligned}
$$

Thus we reject the hypothesis that the reduced model is correct if $F>F_{q-p, n-p}^{(\alpha)}$.

Finally, in order to control for Type I errors, a correction for multiple comparison was performed using the method of clustersize thresholding (implemented in BrainVoyager QX), which has been described in detail elsewhere [15].

\section{Results}

The demographic and personality characteristics of the sample are listed in Table 1. ANOVA did not re- veal significant differences as for age and educational level between the two subgroups $\left(F_{9,3}=1.0\right.$; $P<0.44)$. Mean scores for all considered MMPI2 subscale were higher for the high-psychotic group vs. the low-psychotic group (PA $=47.67$ vs. $42.86 ; \mathrm{PD}$ $=55.50$ vs. $49.29 ; \mathrm{SCF}=41.00$ vs. 30.86 ; respectively); however, only the t-scores for SCF were marginal different $[t(11)=2.237 ; P=0.047]$.

The results of the voxel-based GLM analysis of FA values within the white matter revealed statistically significant differences between the low-psychotic and the high-psychotic group in 51 white matter clusters, as depicted in Fig. 2. The FA values of these significant clusters are summarized on Table 2. In order to characterize the white matter fibers anatomy, a comparison with anatomy atlases and DTI/MRI anatomical images [29] was done; in Fig. 3, all relevant brain

Fig. 2 Voxel-based statistical mapping of significant differences $(P<0.05$, corrected) of FA between subjects with high (High-P) and low (Low-P) scores for psychotic personality traits; sagittal slices are presented from right to left (first slice, $X=50$; last slice, $X=-50$ ). Color scale represents values of $Z$ scores (red coded scale $=$ high psychotic personality traits FA > low psychotic personality $\mathrm{FA}$; blue coded scale $=$ low psychotic personality traits $F A>$ high psychotic personality traits $\mathrm{FA}$ ); coronal and axial projection of the location of sagittal slices are also shown

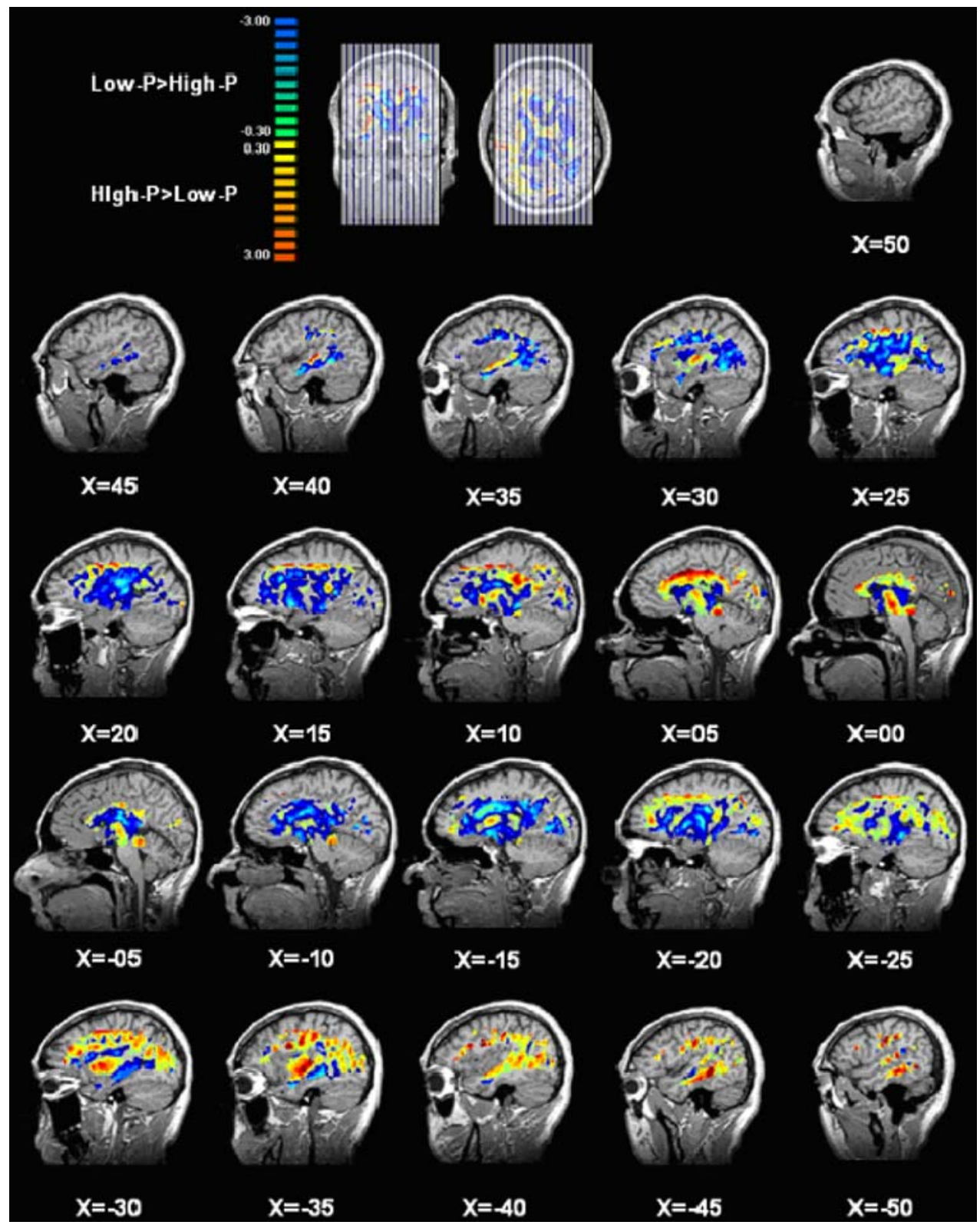




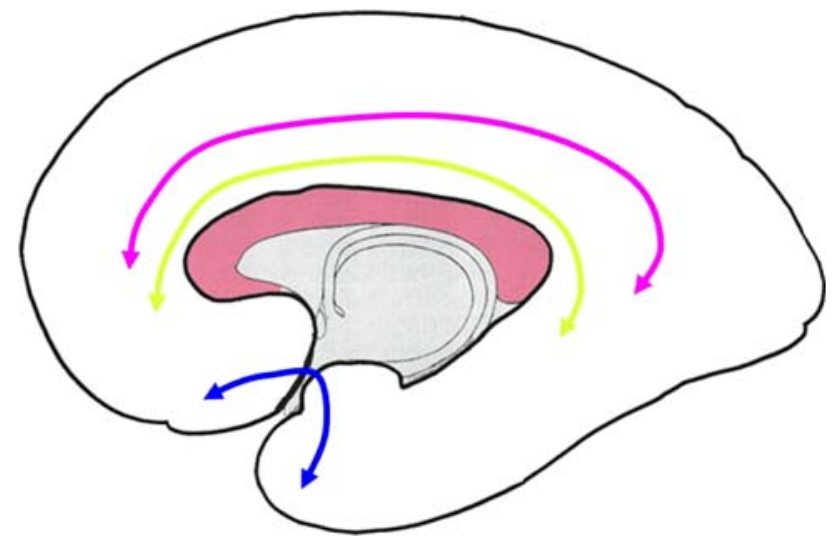

Fig. 3 Schematic representation of major associative white matter fibers in the brain (red $=$ corpus callosum; pink $=$ arcuate faciculus; blue $=$ uncinate fasciculus; green = longitudinal fronto-temporal bundles)

associative tracts are schematically depicted. In 23 clusters within the white matter the FA values were higher for the high-psychotic group as compared to the low-psychotic group, and those clusters corresponded to the fibers belonging to the left arcuate fasciculus. In 28 clusters within the white matter (including fibers of the right arcuate fasciculus, fronto-parieto-temporal fibers and corpus callosum), the FA values were higher for the low-psychotic group as compared to the high-psychotic group.

The subsequent reduced GLM assuming that only the MMPI-2 Sc, $\mathrm{Pa}$ and $\mathrm{Pd}$ scales ratings are predictors of FA values revealed significant effects in all 51 clusters. The averaged amount of variance explained by this model within all clusters was $0.61 \pm 0.09$. The full GLM assuming FA values being predicted by MMPI-2 Sc, Pa and Pd scales values, and by sex and age, revealed significant effects in all clusters. The averaged amount of variance explained by the full model within all clusters was $0.66 \pm 0.09$; it is only $4.2 \%$ higher with respect to that of the reduced model. The reduced and full GLM were tested using the sum of the square of residuals of the two GLMs by F-test. None of the 51 regions of interest showed significant differences between the two models.

A distribution of the FA values for each of the 51 significant clusters separated for male and female and for the high-psychotic and low-psychotic groups are displayed on Fig. 4 (these FA values are extracted from Table 2). This distribution shows that the FA values of males and females in all significant clusters are indistinguishable, providing further evidence, that the sex effect is not a factor of significant influence.

\section{Discussion}

According to our findings, subjects with high scores for psychotic personality traits significantly differ from those with low scores in brain connectivity patterns: in fact, the former group, as compared to the latter one, showed lower inter-hemispheric and right fronto-temporal anatomical connectivity, as well as higher FA values in the left arcuate fasciculus.

In the last decades, many neuropsychological models of schizophrenia spectrum disorders proposed that they should be regarded mainly as "misconnection syndromes". This hypothesis considers psychotic symptoms as the result of a failure of functional integration within the brain. Indeed, brain imaging studies, focusing on brain connectivity in schizophrenia, reported reduced white matter integrity in various brain regions [8]. Our finding of reduced callosal FA in the high versus the low psychotic
Fig. 4 Distribution of global FA values for males and females for all observed 51 significant clusters (the FA values are extracted from Table 2). First group of four columns represent clusters with significantly higher FA values in the group with high scores, as compared to those of the low scores for psychotic personality traits. Second group of four columns represent cluster with significantly higher $F A$ values in the group with low scores, as compared to those with high scores, for psychotic personality traits. No significant difference in $\mathrm{FA}$ values between male (black) and female (light gray) subjects was found

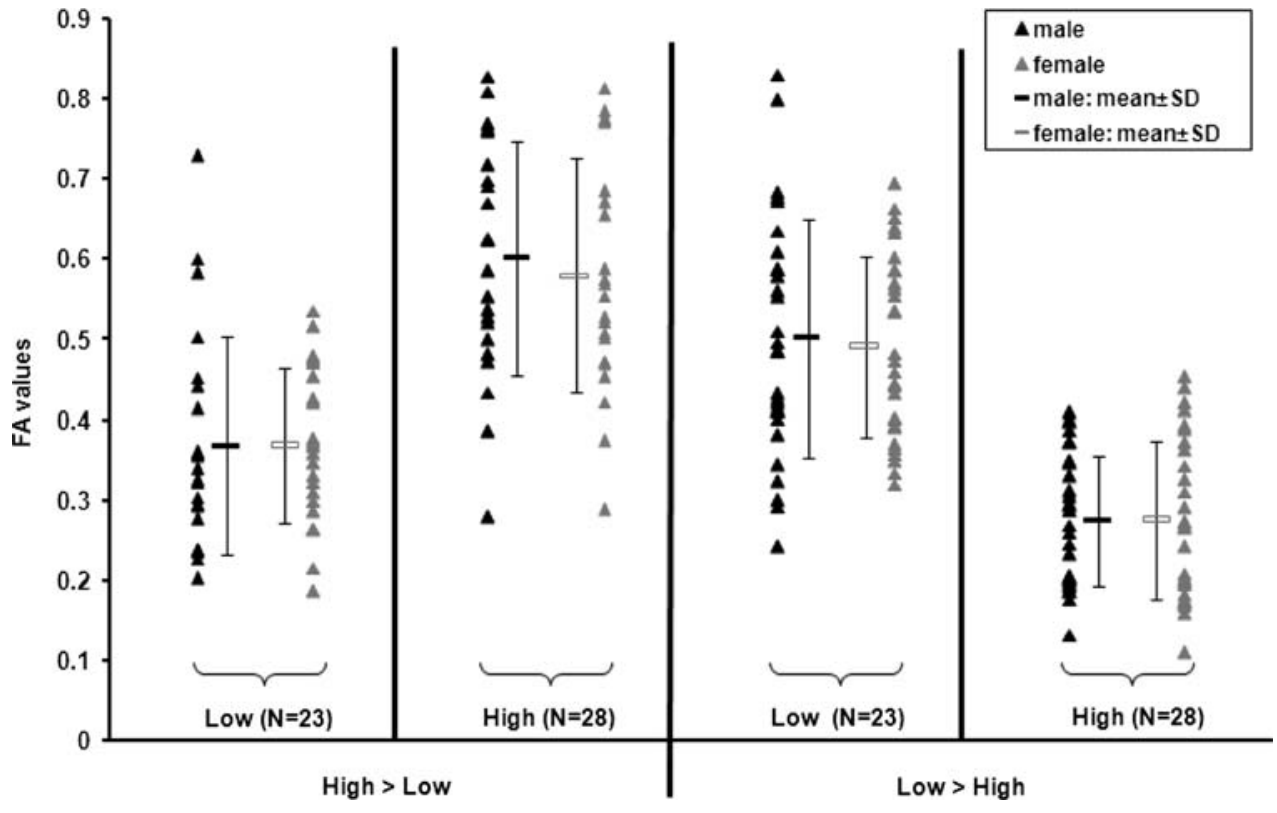


group is in line with the results of other brain imaging studies which reported an inter-hemispheric misconnection (for a review, see [8]), the disruption of corpus callosum microstructure and the worsening of the efficiency of inter-hemispheric processing in schizophrenia $[12,24]$. In the same vein, Downhill et al. [13] in a recent MRI study reported a psychosiscorrelated decrease in callosal bundle, which had the largest size in healthy controls, smaller in schizotypal patients and smallest in schizophrenics.

We also found a different pattern of hemispheric asymmetry in the two subgroups: subjects with higher psychotic scores had higher FA values in the left and lower FA values in the right arcuate fasciculus, as compared to subjects with low scores. A recent DTI study [32], investigating structural connectivity patterns in subjects with schizotypal personality disorder, found a significant reduction of FA and mean diffusivity in the uncinate fasciculus; the FA decrease in the right hemisphere was significantly correlated with clinical symptoms, whereas the same abnormality in the left hemisphere was correlated with measures of cognitive functioning.

Abnormalities in brain hemispheric asymmetries in schizophrenia were repeatedly reported by neuropsychological, neurophysiological and brain imaging studies [19]. Recent DTI findings confirmed the presence of hemisphere asymmetry abnormalities in schizophrenia, mainly involving frontal and temporal lobes: Hubl et al. [20] recently reported higher FA values in the left arcuate fasciculus, in hallucinating schizophrenic patients with respect to both healthy controls and schizophrenic non-hallucinating patients; a recent report by the same group also highlighted that asymmetrical cerebral connectivity abnormalities are present since the first psychotic episode [14]. Park et al. [34], in a recent DTI study, described an attenuated cerebral asymmetry (right > left) pattern in corpus callosum, cingulate, uncinate and superior longitudinal fasciculi, in subjects with schizophrenia, when compared to healthy controls. These findings are in line with our results and further support the hypothesis that differences in brain asymmetry pattern relevant to psychotic features of schizophrenia spectrum.

Psychopathologists have long struggled to differentiate psychotic personality traits from "true" mental illnesses, on the ground that only in the formers the mental state remains normal; however, recent phenomenological and neurobiological models of psychoses tend to accommodate psychiatric disorders, personality disorders and psychotic personality traits in otherwise normal subjects within a single explanatory framework, accounting for genetic, neurodevelopmental and cognitive risk factors [4]. Our data seem to provide further confirmation to such a model, since we found, in a sample of healthy subjects, an abnormal connectivity pattern, which was already reported in schizophrenic samples.
The low number of subjects included in the study and the unbalanced sex ratio between the two subgroups could limit the interpretation of our data, although two different statistical analyses excluded a significant impact of such variables on the reported connectivity differences. Moreover, the locations of differences in FA values between groups are subject to great variation, also depending on the size of the applied Gaussian smoothing kernel. However, in Fig. 2, we show the cluster of significant differences of FA values in both groups as well as the regions that presumably belong to tracts of similar direction. In fact, the t-value threshold scale in this Fig. 2 is ranging from \pm 0.3 to \pm 3 .0. If our results were representing arbitrary locations, depending on the used filter size, we would expect to find (1) significant spots randomly spread over the whole brain region and (2) spots representing group differences of FA values random in its sign. However, we were not able not cover the whole brain with our measurement. In fact, the FA space did only include the talairach space $[-31<z<46]$. We expected to find the majority of putative differences within this brain space, a brain space which in its size was similar to the one used in a previously published DTI study by our group [20]. In spite of the above possible shortcomings, the use of DTI allowed us to detect brain connectivity abnormalities in subjects with psychotic traits, which may be relevant to further understanding of the vulnerability to endogenous psychoses in humans.

\section{Conclusions}

We observed that the presence of psychotic personality traits was correlated to brain connectivity abnormalities in the absence of any overt psychotic manifestation. More in detail, subjects with psychotic traits showed an impaired fronto-temporal connectivity and also a different inter-hemispheric connectivity pattern, as compared to subjects with low scores. This evidence may be relevant for the comprehension of the brain structural and functional abnormalities in schizophrenia spectrum disorders.

\section{References}

1. Alfimova MV, Trubnikov VI, Orlova VA (1995) Inheritability of personality traits measured by MMPI in families of schizophrenic patients. Genetika 31:1010-1015

2. Basser PJ, Pierpaoli C (1998) A simplified method to measure the diffusion tensor from seven MR images. Magn Reson Med 39:928-934

3. Basser PJ, Mattiello J, LeBihan D (1994) Estimation of the effective self-diffusion tensor from the NMR spin echo. J Magn Reson B 103:247-254 
4. Bentall R (2006) Madness explained: why we must reject the Kraepelinian paradigm and replace it with a 'complaint-orientated' approach to understanding mental illness. Med Hypotheses 66:220-233

5. Berry DT, Wetter MW, Baer RA, Widiger TA, Sumpter JC, Reynolds SK, Hallam RA (1991) Detection of random responding on the MMPI-2: utility of F, Back F, and VRIN scales. Psychol Assess 3:418-423

6. Bleuler E (1924) Textbook of psychiatry. Macmillan, New York

7. Bolinskey PK, Gottesman II, Nichols DS, Shapiro BM, Roberts SA, Adamo UH, Erlenmeyer-Kimling L (2001) A new MMPIderived indicator of liability to develop schizophrenia: evidence from the New York High-Risk Project. Assessment $8: 127-143$

8. Brambilla P, Cerini R, Gasparini A, Versace A, Andreone N, Vittorini E, Barbui C, Pelizza L, Nose M, Barlocco L, Marrella G, Gregis M, Tournikioti K, David AS, Keshavan MS, Tansella M (2005) Investigation of corpus callosum in schizophrenia with diffusion imaging. Schizophr Res 79:201-210

9. Carter JW, Parnas J, Cannon TD, Schulsinger F, Mednick SA (1999) MMPI variables predictive of schizophrenia in the Copenhagen High-Risk Project: a 25-year follow-up. Acta Psychiatr Scand 99:432-440

10. Catani M (2006) Diffusion tensor magnetic resonance imaging tractography in cognitive disorders. Curr Opin Neurol 19:599606

11. Dahlstrom WG, Welsh GS, Dahlstrom LE (1972) An MMPI handbook. Clinical interpretation, vol I. University of Minnesota Press, Minneapolis

12. Diwadkar VA, DeBellis MD, Sweeney JA, Pettegrew JW, Keshavan MS (2004) Abnormalities in MRI-measured signal intensity in the corpus callosum in schizophrenia. Schizophr Res $67: 277-282$

13. Downhill JE Jr, Buchsbaum MS, Wei T, Spiegel-Cohen J, Hazlett EA, Haznedar MM, Silverman J, Siever LJ (2000) Shape and size of the corpus callosum in schizophrenia and schizotypal personality disorder. Schizophr Res 42:193-208

14. Federspiel A, Begre S, Kiefer C, Schroth G, Strik WK, Dierks T (2006) Alterations of white matter connectivity in first episode schizophrenia. Neurobiol Dis 22:702-709

15. Forman SD, Cohen JD, Fitzgerald M, Eddy WF, Mintun MA, Noll DC (1995) Improved assessment of significant activation in functional magnetic resonance imaging (fMRI): use of a cluster-size threshold. Magn Reson Med 33:636-647

16. Friston KJ (1999) Schizophrenia and the disconnection hypothesis. Acta Psychiatr Scand Suppl 395:68-79

17. Frith C (2005) The neural basis of hallucinations and delusions. C R Biol 328:169-175

18. Graham JR (1974) The MMPI: a practical guide, 2nd edn. Oxford University Press, Oxford

19. Gur RE, Chin S (1999) Laterality in functional brain imaging studies of schizophrenia. Schizophr Bull 25:141-156

20. Hubl D, Koenig T, Strik W, Federspiel A, Kreis R, Boesch C, Maier SE, Schroth G, Lovblad K, Dierks T (2004) Pathways that make voices: white matter changes in auditory hallucinations. Arch Gen Psychiatry 61:658-668
21. Johns LC, van Os J (2001) The continuity of psychotic experiences in the general population. Clin Psychol Rev 21:1125-1141

22. Jones DK, Symms MR, Cercignani M, Howard RJ (2005) The effect of filter size on VBM analyses of DT-MRI data. Neuroimage 26:546-554

23. Kanaan RA, Kim JS, Kaufmann WE, Pearlson GD, Barker GJ, McGuire PK (2005) Diffusion tensor imaging in schizophrenia. Biol Psychiatry 58:921-929

24. Kasai K, McCarley RW, Salisbury DF, Onitsuka T, Demeo S, Yurgelun-Todd D, Kikinis R, Jolesz FA, Shenton ME (2004) Cavum septi pellucidi in first-episode schizophrenia and firstepisode affective psychosis: an MRI study. Schizophr Res 71:65-76

25. Kraepelin E (1919) Dementia praecox and paraphrenia. Krieger, New York

26. Kretschmer E (1934) A textbook of medical psychology. Oxford University Press, Oxford

27. Lachar D (1974) The MMPI: clinical assessment and automated interpretation. Western Psychological Services, Los Angeles

28. Maj M (1998) Critique of the DSM-IV operational diagnostic criteria for schizophrenia. Br J Psychiatry 172:458, 460

29. Mamata H, Mamata Y, Westin CF, Shenton ME, Kikinis R, Jolesz FA, Maier SE (2002) High-resolution line scan diffusion tensor MR imaging of white matter fiber tract anatomy. AJNR Am J Neuroradiol 23:67-75

30. Meehl PE (1989) Schizotaxia revisited. Arch Gen Psychiatry 46:935-944

31. Mucci A, Galderisi S, Bucci P, Tresca E, Forte A, Koenig T, Maj M (2005) Hemispheric lateralization patterns and psychotic experiences in healthy subjects. Psychiatry Res 139:141-154

32. Nakamura M, McCarley RW, Kubicki M, Dickey CC, Niznikiewicz MA, Voglmaier MM, Seidman LJ, Maier SE, Westin CF, Kikinis R, Shenton ME (2005) Fronto-temporal disconnectivity in schizotypal personality disorder: a diffusion tensor imaging study. Biol Psychiatry 58:468-478

33. Oldfield RC (1971) The assessment and analysis of handedness: the Edinburgh inventory. Neuropsychologia 9:97-113

34. Park HJ, Westin CF, Kubicki M, Maier SE, Niznikiewicz M, Baer A, Frumin M, Kikinis R, Jolesz FA, McCarley RW, Shenton ME (2004) White matter hemisphere asymmetries in healthy subjects and in schizophrenia: a diffusion tensor MRI study. Neuroimage 23:213-223

35. Rossler W, Riecher-Rossler A, Angst J, Murray R, Gamma A, Eich D, van Os J, Gross VA (2007) Psychotic experiences in the general population: a twenty-year prospective community study. Schizophr Res 92:1-14

36. Shergill SS, Kanaan RA, Chitnis XA, O'Daly O, Jones DK, Frangou S, Williams SC, Howard RJ, Barker GJ, Murray RM, McGuire P (2007) A diffusion tensor imaging study of fasciculi in schizophrenia. Am J Psychiatry 164:467-473

37. Subotnik KL, Nuechterlein KH, Green MF (1999) Trait versus state aspects of the MMPI during the early course of schizophrenia. J Psychiatr Res 33:275-284

38. Wetzler S, Khadivi A, Moser RK (1998) The use of the MMPI-2 for the assessment of depressive and psychotic disorders. Assessment 5:249-261 